\title{
Sox 7 controls arterial specification in conjunction with hey2 and efnb2 function
}

\author{
Dorien M. A. Hermkens ${ }^{1,2}$, Andreas van Impel ${ }^{1}$, Akihiro Urasaki ${ }^{1}$, Jeroen Bussmann ${ }^{1}$, Henricus J. Duckers ${ }^{2}$ and \\ Stefan Schulte-Merker ${ }^{1,3,4, *}$
}

\begin{abstract}
SoxF family members have been linked to arterio-venous specification events and human pathological conditions, but in contrast to Sox17 and Sox18, a detailed in vivo analysis of a Sox7 mutant model is still lacking. In this study we generated zebrafish sox7 mutants to understand the role of Sox7 during vascular development. By in vivo imaging of transgenic zebrafish lines we show that sox7 mutants display a short circulatory loop around the heart as a result of aberrant connections between the lateral dorsal aorta (LDA) and either the venous primary head sinus (PHS) or the common cardinal vein (CCV). In situ hybridization and live observations in flt4:mCitrine transgenic embryos revealed increased expression levels of flt4 in arterial endothelial cells at the exact location of the aberrant vascular connections in sox 7 mutants. An identical circulatory short loop could also be observed in newly generated mutants for hey2 and efnb2. By genetically modulating levels of sox7, hey2 and efnb2 we demonstrate a genetic interaction of sox 7 with hey2 and efnb2. The specific spatially confined effect of loss of Sox7 function can be rescued by overexpressing the Notch intracellular domain (NICD) in arterial cells of sox 7 mutants, placing Sox7 upstream of Notch in this aspect of arterial development. Hence, sox 7 levels are crucial in arterial specification in conjunction with hey2 and efnb2 function, with mutants in all three genes displaying shunt formation and an arterial block.
\end{abstract}

KEY WORDS: Vascular development, Arterial-venous specification, Sox7, Zebrafish

\section{INTRODUCTION}

One of the first organs that develop in the vertebrate body is the vascular system. Abnormalities in vascular development can cause endothelial malformations ranging from severe birth defects to mild lesions (Brouillard and Vikkula, 2007). The vascular system consists of endothelial cells (ECs) that become specified into arterial, venous and lymphatic cells, eventually forming a functional vascular system. Vascular development starts with the migration of mesodermal-derived angioblasts, which in zebrafish are localized in two bilaterally positioned populations in the lateral plate mesoderm. It was recently reported that medial angioblasts start migrating around $14 \mathrm{hpf}(10$-somite stage) and the lateral angioblasts initiate

\footnotetext{
${ }^{1}$ Hubrecht Institute - Royal Netherlands Academy of Arts and Sciences and University Medical Centre Utrecht, Uppsalalaan 8, 3584 CT Utrecht, The Netherlands. ${ }^{2}$ Erasmus MC Rotterdam, 's-Gravendijkwal 230, 3015 CE Rotterdam, The Netherlands. ${ }^{3}$ Cells-in-Motion Cluster of Excellence (EXC 1003 - CiM), University of Münster, 48149 Münster, Germany. ${ }^{4}$ Institute for Cardiovascular Organogenesis and Regeneration, Faculty of Medicine, Westfälische WilhelmsUniversität Münster (WWU), Mendelstrasse 7, 48149 Münster, Germany.

*Author for correspondence (schultes@ukmuenster.de)
}

Received 1 September 2014; Accepted 6 March 2015 migration to the midline around $16 \mathrm{hpf}$ (15-somite stage). The medially located angioblasts will form the arterial cells of the first arterial vessel, the dorsal aorta (DA), and the laterally localized angioblasts will give rise exclusively to the venous cells of the first venous vessel: the posterior cardinal vein (PCV) (Zhong et al., 2000; Kohli et al., 2013). Vascular endothelial growth factor (Vegf) receptors and their ligands play crucial roles during arterial-venous specification. Vegf receptor 3/Flt4 is initially expressed in all ECs and becomes restricted to venous and lymphatic ECs later in development (Kaipainen et al., 1995; Hogan et al., 2009b; van Impel et al., 2014). Inactivation of Vegfr3 in mice has been shown to result in decreased angiogenic sprouting and vascular network formation (Tammela et al., 2008). Activation of Vegf receptor 2, also expressed in ECs, by VegfA and Sonic Hedgehog (Shh) signaling induces the PLC $\gamma / \mathrm{Mek} /$ Erk pathway and subsequently the Notch signaling pathway (Lawson et al., 2002). Upon activation of the Notch receptor by binding to one of its ligands (Delta-like, Jagged), the Notch intracellular domain (NICD) is released from the plasma membrane via proteolytic processing. The NICD translocates to the nucleus where it can bind to Suppressor of Hairless $[\mathrm{Su}(\mathrm{H})]$. This complex can mediate transcription of Hairy/ Enhancer of Split (Hes) and Hes-related genes (Hey/HRT/HERP) and the expression of ephrinB2 (efnb2) on the arterial membrane (Lawson et al., 2001; Zhong et al., 2001). Efnb2 is a member of the Ephrin family and is a largely arterial-specific transmembrane protein that functions as a ligand for the venous receptor tyrosine kinase Eph receptor B4 (Ephb4). Signaling requires cell-to-cell contact and can be bidirectional. The reciprocal signaling between Efnb2 and Ephb4 is crucial in arterial-venous specification (Wang et al., 1998; Gerety et al., 1999).

Previous work has suggested a role for the SRY-related HMG box (Sox) gene family in various aspects of vascular development (reviewed by Francois et al., 2010). The sox gene family encodes transcription factors and consists of 10 subgroups (SoxA-J). All members of the Sox family contain a high mobility group box (HMG) domain, which facilitates DNA binding in the minor groove and mediates DNA bending (Giese et al., 1992), and contain a transactivation domain (TAD), which activates transcription of target genes (Hosking et al., 1995). One subgroup of the Sox family that is of particular interest for vascular development is the SoxF group, consisting of SOX7, SOX17 and SOX18. In vitro studies revealed that SoxF transcription factors can bind the arterial-specific enhancer of the Notch ligand dll4 (Sacilotto et al., 2013). Furthermore, Sox 17 has recently been shown to play a key role in endoderm formation, hematopoietic stem cell regulation and the acquisition of arterial identity by functioning upstream of Notch signaling (Hudson et al., 1997; Kanai-Azuma et al., 2002; He et al., 2011; Corada et al., 2013). Mutations in SOX18 are linked to the human hypotrichosis-lymphedema-telangiectasia (HLT) syndrome, in which individuals have severe lymphedema, vascular leakages 
and disrupted hair follicle development (Irrthum et al., 2003). The phenotype of mice with a truncated Sox 18 protein (mutation in the ragged opossum allele) resembles this syndrome, resulting in severe edema, blood vessel disruption and early lethality (Pennisi et al., 2000; James et al., 2003). On some genetic backgrounds in mice, Sox 18 is required for the differentiation of ECs in lymphatic cells by initiating expression of Prox 1 (François et al., 2008). Recent work in zebrafish, however, has shown that sox18 is dispensable for lymphatic specification in the fish (van Impel et al., 2014). A simultaneous knockdown of sox 7 and sox 18 transcripts in zebrafish results in disruption of arterial-venous segregation at $48 \mathrm{hpf}$, followed by shunt formation between the DA and the PCV. sox7/ sox 18 double morphants display an increase in venous markers and a decrease of arterial markers in the DA, corroborating the involvement of Sox7/Sox18 in arterial-venous specification (Cermenati et al., 2008; Herpers et al., 2008; Pendeville et al., 2008). Previous reports in zebrafish could not identify a specific vascular function for Sox 7, though these studies were limited by the use of morpholinos (Cermenati et al., 2008; Herpers et al., 2008; Pendeville et al., 2008). Mice lacking Sox7 die at embryonic day 10.5 due to cardiovascular failure (Wat et al., 2012); this early lethality, together with delayed development, pericardial edema and failure of yolk sac remodeling, precludes analysis of underlying cellular mechanisms. To understand the specific role of Sox 7 during vascular development, we therefore generated a zebrafish sox7 mutant. Here, we demonstrate a highly specific arterial-venous shunt phenotype in sox 7 mutants and connect Sox 7 function to Hey2/Notch signaling and to Efnb2 function.

\section{RESULTS}

\section{Blood circulation is perturbed in sox 7 mutant embryos}

Previous observations in zebrafish demonstrated that sox7 is expressed in ECs of the major vessels in the head and trunk, such as the PCV and the (lateral) DA (Cermenati et al., 2008; Herpers et al., 2008; Pendeville et al., 2008) (Fig. 1A). To assess the specific function of Sox7, we generated sox 7 mutants by targeting induced local lesions in genomes (TILLING) (Wienholds et al., 2002). The sox $7^{\text {hu}} 5626$ allele comprises a guanine-to-adenine mutation leading to a predicted premature stop-codon (after amino acid 53) within the HMG domain (Fig. 1B; supplementary material Fig. S1). Sequencing of cDNA from sox $7^{h u 5626}$ mutant embryos did not reveal alternative transcripts lacking the nonsense mutation (supplementary material Fig. S2), suggesting that the sox7 allele represents a loss-of-function situation. The overall appearance of these mutants is normal during early development (Fig. 1C); however, we noticed severe edema formation in mutant embryos from $72 \mathrm{hpf}$ onwards, leading to lethality by day 5 . Analysis of homozygous sox 7 mutants in a kdrl:eGFP;gatal:dsRed transgenic background revealed that the majority ( $59 \%, n=275$ embryos) lack blood circulation in the trunk from the start of circulation onwards

\section{A}

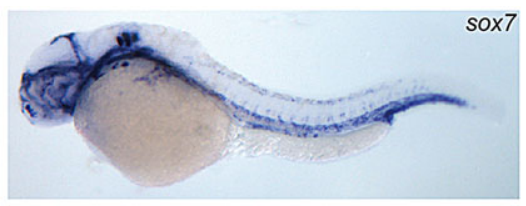

$\mathrm{C}$
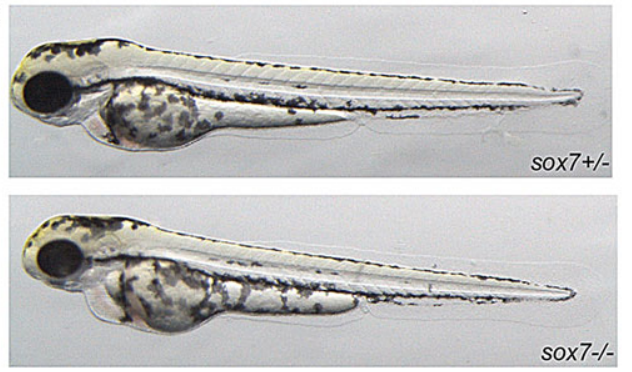
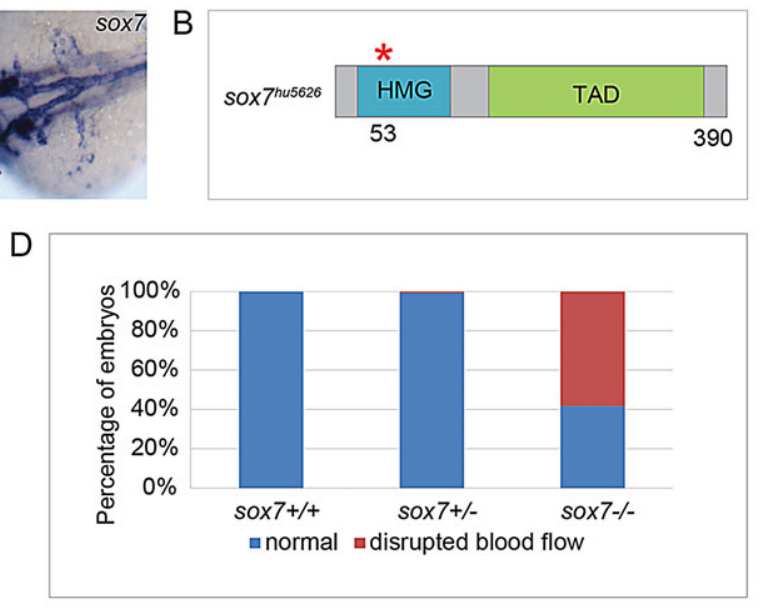

$\mathrm{E}$
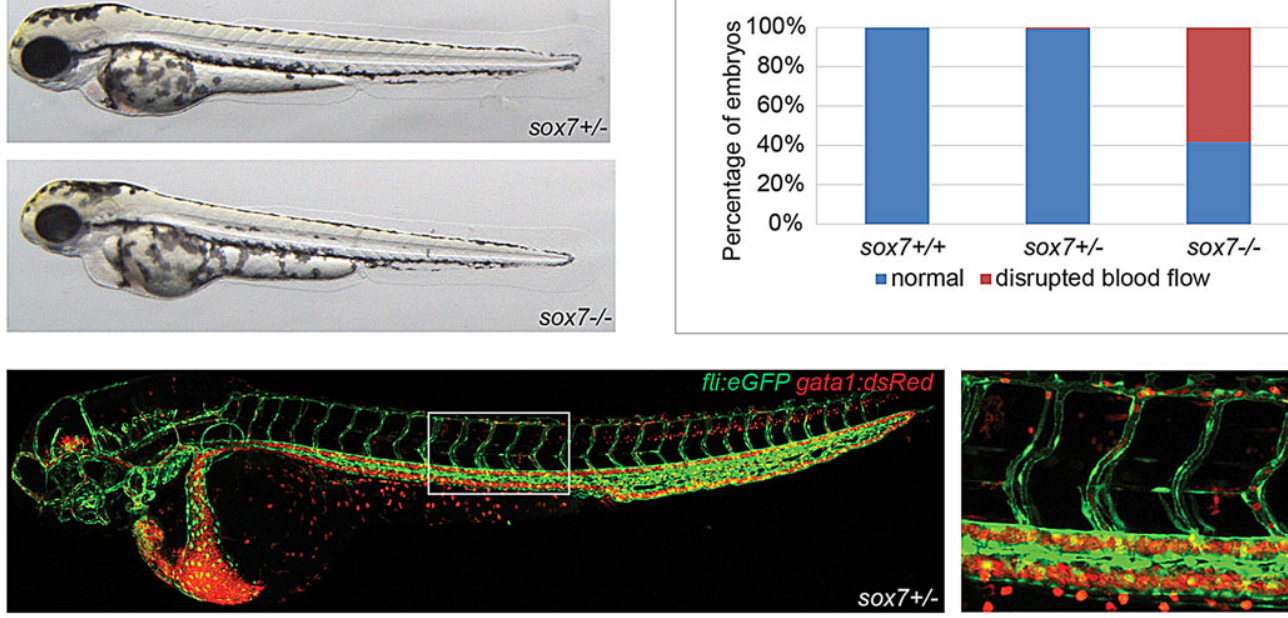

$50 \times 7+/$
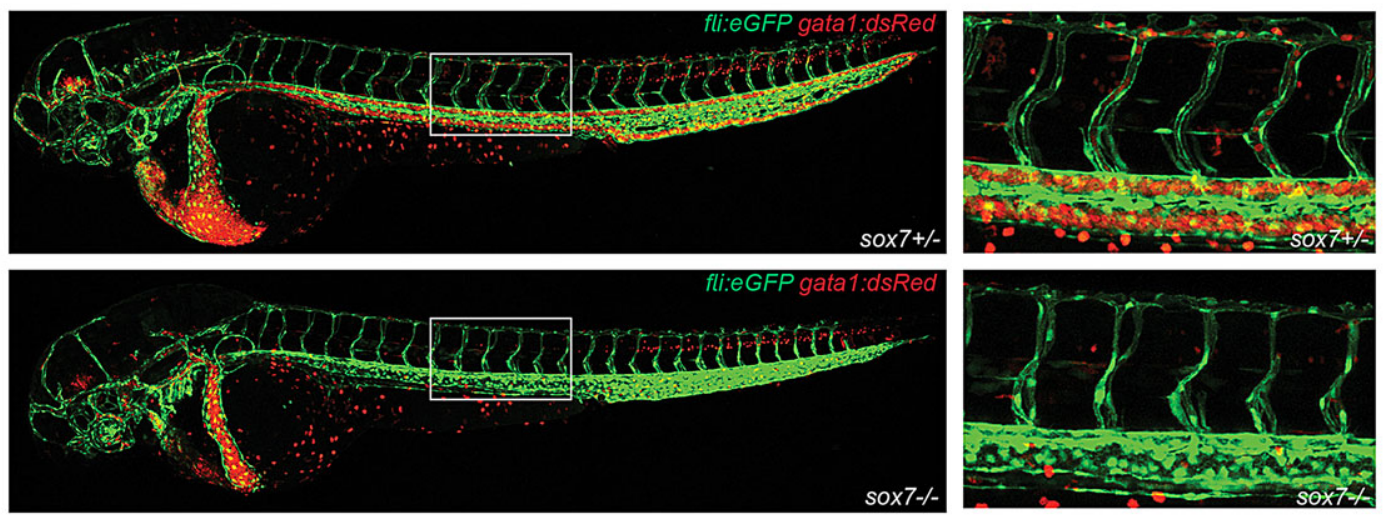

Fig. 1. Disrupted blood circulation in sox7 zebrafish mutants. (A) sox 7 in situ hybridization of 3 dpf wild-type embryos (left panel, lateral view; right panel, dorsal view) showing sox 7 expression in all main vessels. (B) Schematic diagram of the sox $7^{\text {hu5626 }}$ allele with a premature stop-codon after amino acid 53

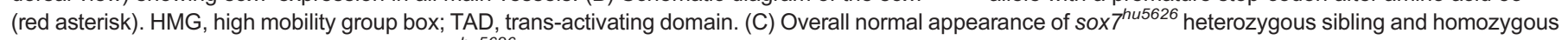
mutant at $2 \mathrm{dpf}$. (D) On average, $59 \%$ of sox $7^{\text {hu5626 }}$ mutants display disturbed blood flow at 2.5 dpf. Percentages of pooled embryos from four independent experiments (total of 275 embryos). Percentages can vary substantially between different backgrounds. (E) kdrl:GFP;gata1:dsRed-positive sox $7^{\text {hu5626 }}$ mutants lack functional blood circulation in the trunk, whereas heterozygous siblings have normal circulation at $2.5 \mathrm{dpf}$. Right panels: higher magnifications of boxed area. 
(Fig. 1D,E). Further analysis in different transgenic backgrounds showed that although this phenotype could always be detected in a fraction of homozygous sox 7 mutants, the actual penetrance of the blood circulatory defect is largely dependent on the genetic background.

When analyzing the blood flow by making use of bright-field movies, we could observe a very specific short loop of circulation in the sox 7 mutants with no circulation in the trunk. This short loop of circulation was never observed in sox 7 siblings (supplementary material Movies 1 and 2).

To characterize the blood circulation defect in $\operatorname{sox} 7$ mutants in more detail, we performed micro-angiographies. In sox 7 mutants, the rhodamine dextran dye distribution can be detected from the injection site within the PCV towards the beating heart; however, we never observed distribution from the heart into the axial DA (Fig. 2A). This indicates complete blockage of blood flow at the
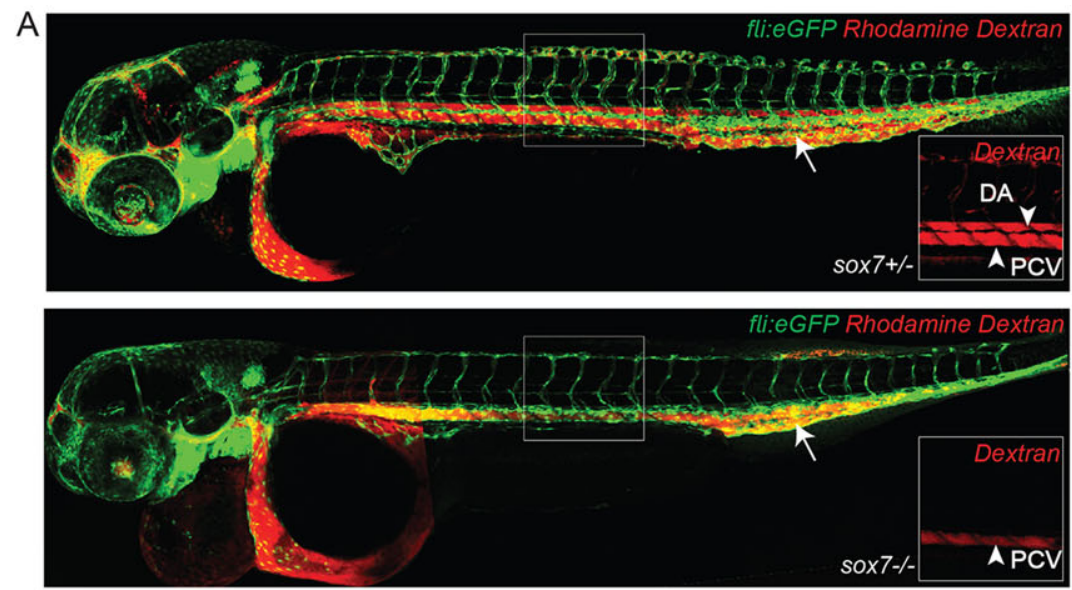

$\mathrm{B}$
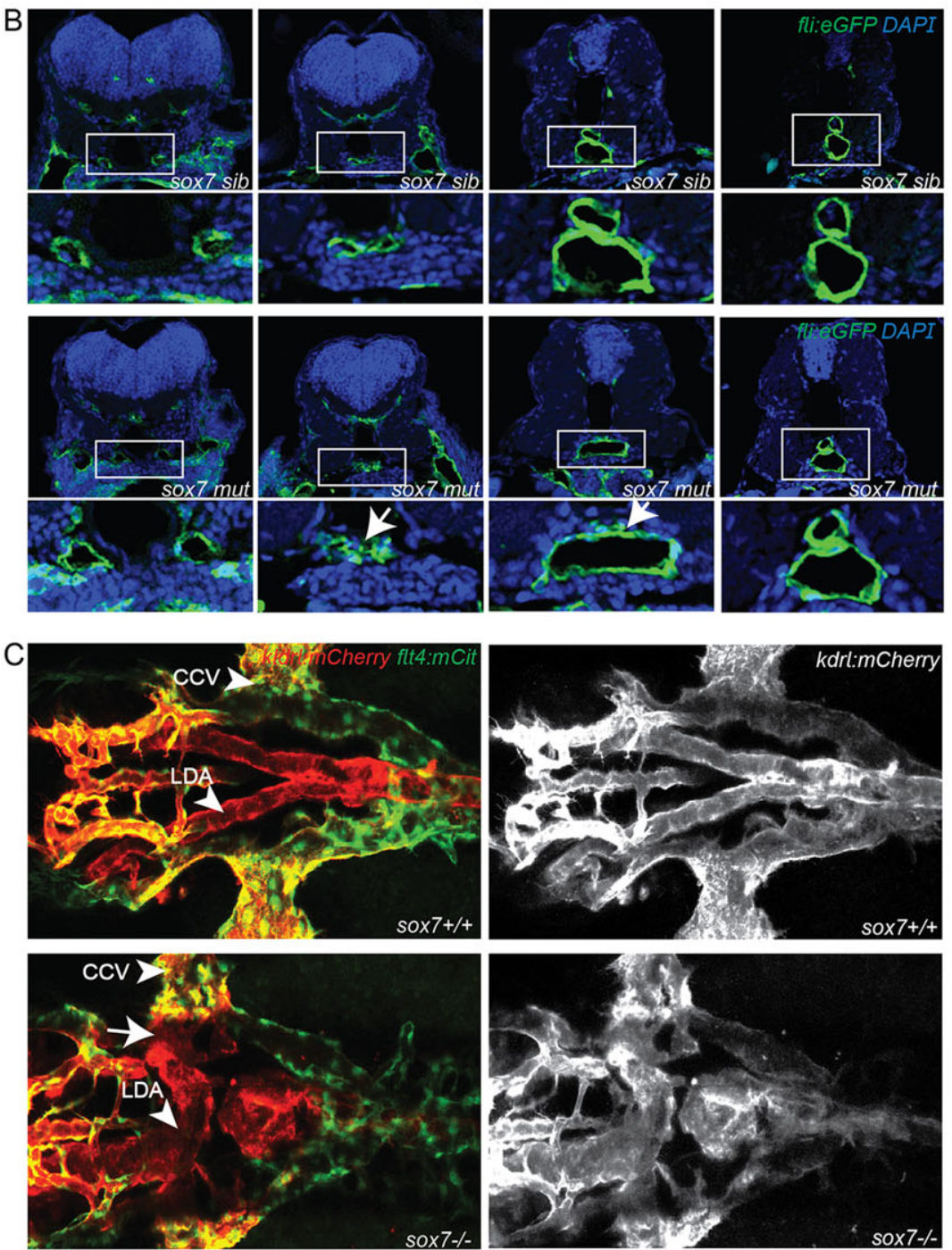

Fig. 2. sox7 mutants show an altered morphology of the LDA while displaying normal DA-PCV segregation in the trunk. (A) Rhodamine-dextran angiograms (injected into the PCV at the position of the arrow) of sox $7^{\text {hu5626 }}$ fli1a:eGFP siblings (upper panel) and mutants (lower panel) at $2 \mathrm{dpf}$. Insets depict the rhodamine-dextran channel of the boxed area with higher magnification highlighting the lack of dye uptake in the DA of sox $7^{\text {hu5626 }}$ mutants. (B) Transverse sections of fli1a:eGFP-positive sox $7^{\text {hu5626 }}$ sibling and mutan embryos at $2 \mathrm{dpf}$, stained with anti-GFP (green) and DAPI (blue). In sox $7^{\text {hu5626 }}$ mutants, the aortic morphology is disturbed at the position of LDA/DA fusion (arrows) while being unaffected more anteriorly and posteriorly to this position. For relative positions of sections, see supplementary material Fig. S3. (C) Dorsal view of sox $7^{\text {hu5626 }}$

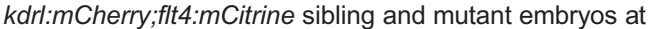
$2.5 \mathrm{dpf}$. Right panel kdrl:mCherry channel only. Shunt formation (arrow) occurs in sox $7^{\text {hu5626 }}$ mutants at the position of LDA fusion. (L) DA, (lateral) dorsal aorta; PCV, posterior cardinal vein; $\mathrm{CCV}$, common cardinal vein 
anterior part of the DA. Cross-sections of $48 \mathrm{hpf}$ sox 7 mutants revealed a locally restricted, disrupted morphology of ECs and defective lumen formation at the position where, in sibling embryos, both lateral dorsal aortae (LDA) fuse to form the dorsal aorta (DA). Both anterior and posterior to this position, the structure of the aorta in mutant embryos appeared to be unaffected (Fig. 2B; supplementary material Fig. S3). Subsequent analysis of sox 7 mutants at $2.5 \mathrm{dpf}$ in $k d r l: m$ Cherry;flt4:mCitrine transgenic embryos, in which we could distinguish arterial and venous ECs, revealed a direct connection between the LDA and the venous primary head sinus (PHS) or the common cardinal vein (CCV) (Fig. 2C). In wild-type embryos, the blood is guided from the bulbus arteriosus (BA) and ventral aorta (VA) into either the LDA and subsequently to the DA in the trunk, or from the VA to the primitive internal carotid artery towards the head region. The blood from the trunk is returned towards the heart by the posterior cardinal vein (PCV) and the CCV, and from the head via the posterior hindbrain channel, the anterior cardinal vein and the primary head sinus (PHS) to the $\mathrm{CCV}$ and the heart. The shunt in sox 7 mutants causes the blood to circulate from the BA and VA into the LDA from where it directly returns via the PHS and CCV to the heart without entering the trunk region. Consistently, ectopic connections between the LDA and the PHS and between the LDA and CCV are evident in sox7 mutants (Fig. 3A,B), while no other abnormalities in the development of the head vasculature could be detected (data not shown).

To investigate the temporal relationship between the blockage of circulation at the fusion site of both LDAs and the shunt formation between LDA and the CCV/PHS, we performed time-lapse imaging of kdrl:eGFP-positive sox7 mutants and siblings starting at the 20somite stage. In wild-type embryos, the LDA is formed by migration of an anterior and a posterior subpopulation of ECs that fuse and give rise to a continuous LDA on both sides of the embryo (Siekmann et al., 2009). In sox 7 mutants, we found that ECs from the developing LDA and the CCV (called Duct of Cuvier at this stage) formed an ectopic connection already during the formation of both vessels and prior to the onset of circulation (Fig. 3C; supplementary material Movies 3 and 4). This finding is consistent with the observation that embryos displaying the shortloop phenotype do not even transiently establish blood flow in the trunk during early stages of development. In conclusion, these results indicate that the shunt formation in $\operatorname{sox} 7$ mutants is not a secondary phenotype caused by the blockage of blood circulation at the fusion site of the two LDAs, but is a specific effect caused by the lack of functional Sox7.

It was previously reported that sox $7 /$ sox 18 double morphants have multiple ectopic connections between the DA and PCV (Cermenati et al., 2008; Herpers et al., 2008; Pendeville et al., 2008). To exclude toxic effects of the combined morpholino knockdown, we generated sox 7; sox 18 double mutants and also found multiple shunts between the DA and PCV in our genetic mutants, thereby fully recapitulating the morphant phenotype (Fig. 3D). However, no ectopic connections between the DA and PCV were observed in sox 7 or sox 18 single mutants (Fig. 3E) (van Impel et al., 2014), reconfirming the strong redundancy between both SoxF transcription factors in the trunk vasculature (Cermenati et al., 2008; Herpers et al., 2008; Pendeville et al., 2008). In addition, we noticed that the penetrance of the sox $7^{-/-}$short loop phenotype was enhanced by the additional loss of one copy of sox 18, whereas homozygous sox 18 mutants lacking one copy of sox7 did not establish the circulatory short loop at all (Fig. 3D; data not shown). These results suggest a compensatory role for Sox 18 in the absence of Sox7 in the LDA; however, they also emphasize the highly specific local requirement of normal Sox7 levels in this specific part of the aorta.

To explore the involvement of the third SoxF family member, Sox17, in the context of LDA development, we analyzed its expression pattern by employing a sox 17:eGFP transgenic reporter line (Mizoguchi et al., 2008). Although sox17 is expressed in arterial cells of the dorsal aorta and ISVs (supplementary material Fig. S4A), we could not detect sox 17:eGFP expression in the LDA of sox7 siblings or mutants (supplementary material Fig. S4B). Furthermore, the overall sox17:eGFP expression levels in the arterial ECs of sox 7 siblings and mutants are indistinguishable at 1 and $2 \mathrm{dpf}$ (supplementary material Fig. S4A; data not shown). Therefore, although we cannot fully exclude an involvement of Sox17 in some aspect of LDA development, our analysis suggests that $\operatorname{sox} 17$ is not expressed at the right stages within the LDA to affect its formation, unlike sox7 and sox 18 , the mRNAs of which can readily be detected in ECs of this specific vascular structure.

\section{fms-related tyrosine kinase 4 (flt4) expression is altered in arterial cells of sox 7 mutants}

To assess the involvement of sox 7 in vascular development, we performed whole-mount in situ hybridization for arterial and venous specific genes. This revealed expression of flt4, also called vegfr 3 , to be upregulated in arterial cells at the location of ectopic arteriovenous connections in most sox 7 mutants at $20 \mathrm{hpf}$ (Fig. 4A,D). Vegfr3/Flt4 is a transmembrane tyrosine kinase receptor for the ligands VegfC and VegfD, and becomes quickly restricted to only venous and lymphatic endothelial cells (Kaipainen et al., 1995; Joukov et al., 1996; Achen et al., 1998; Hogan et al., 2009b; van Impel et al., 2014). We confirmed our observation of induced flt4 expression with a transgenic reporter line and found flt4:mCitrine expression to be specifically increased in the LDA of sox 7 mutants at $26 \mathrm{hpf}$, compared with the expression in the LDA of wild-type embryos at this time point (Fig. 4B). Expression of flt4 was also increased in the DA and ISVs in 58\% of sox7 mutants at $26 \mathrm{hpf}$ (Fig. 4C,E). Although the ectopic expression of this venous marker would suggest differential expression of other arterial and/or venous markers, we did not detect this for a wide range of other marker genes (notch1b, notch3, efnb2, hey2, dll4, foxc1, sox 18, dab2, nr2f2 and ephb4) in sox 7 mutants at several time points (20-30 hpf) (Fig. 4A,C; data not shown).

\section{efnb2 and hey2 genetically interact with sox7}

The spatially confined defect in sox 7 mutants is a rather unique phenotype; however, we serendipitously observed identical defects in double mutants for efnb2a and efnb2b. Both efnb2 mutants were generated by TILLING, and contain point-mutations leading to predicted premature stop-codons (after amino acid 86 in efnb2 $a^{h u 3393}$ and amino acid 78 in efnb2 $b^{h u 2971}$ ) (Fig. 5A). We found that efn $b 2 a$ as well as $e f n b 2 b$ single mutants do not develop any obvious vascular defects; however, simultaneous loss of both genes resulted in a significant increase of circulatory short-circuits in $42 \%$ of double mutants (Fig. 5B,C). As sox 7 mutants also show only a partially penetrant phenotype, this allowed us to investigate a possible connection between sox 7 and efnb2 genes by testing for genetic interactions and hence we generated triple mutants. Importantly, increasing the number of loss-of-function alleles was accompanied by an increase in the amount of embryos displaying the short-loop phenotype. Furthermore, while all efnb2a single mutants had normal circulation, and while only $17 \%$ of sox 7 mutants exhibited the short-loop phenotype in this 
A
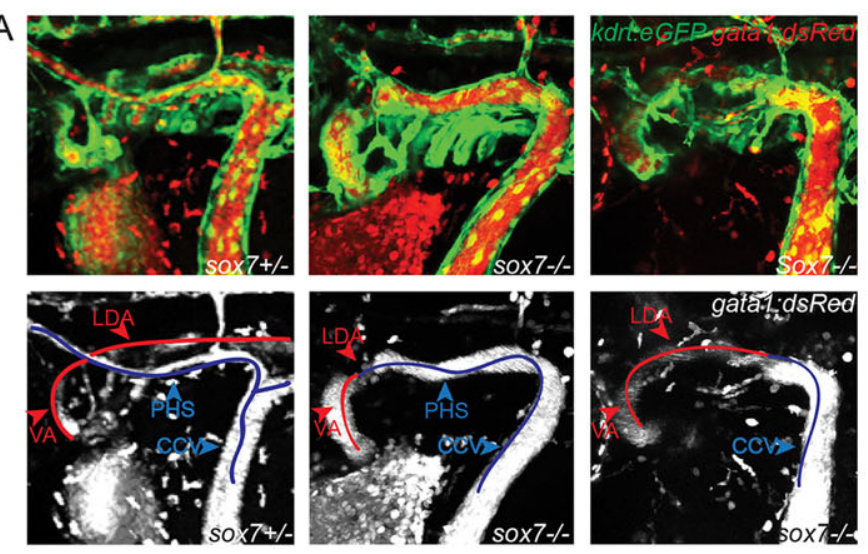

B

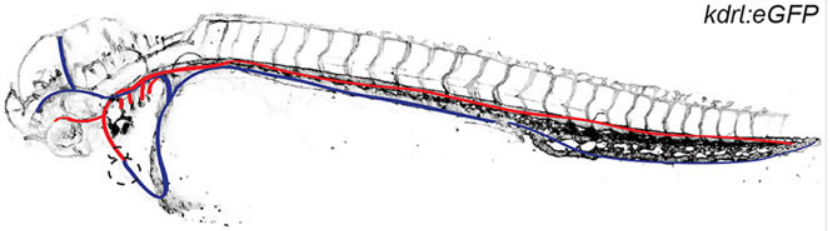

sox $7+/+$

kdrl:eGFP
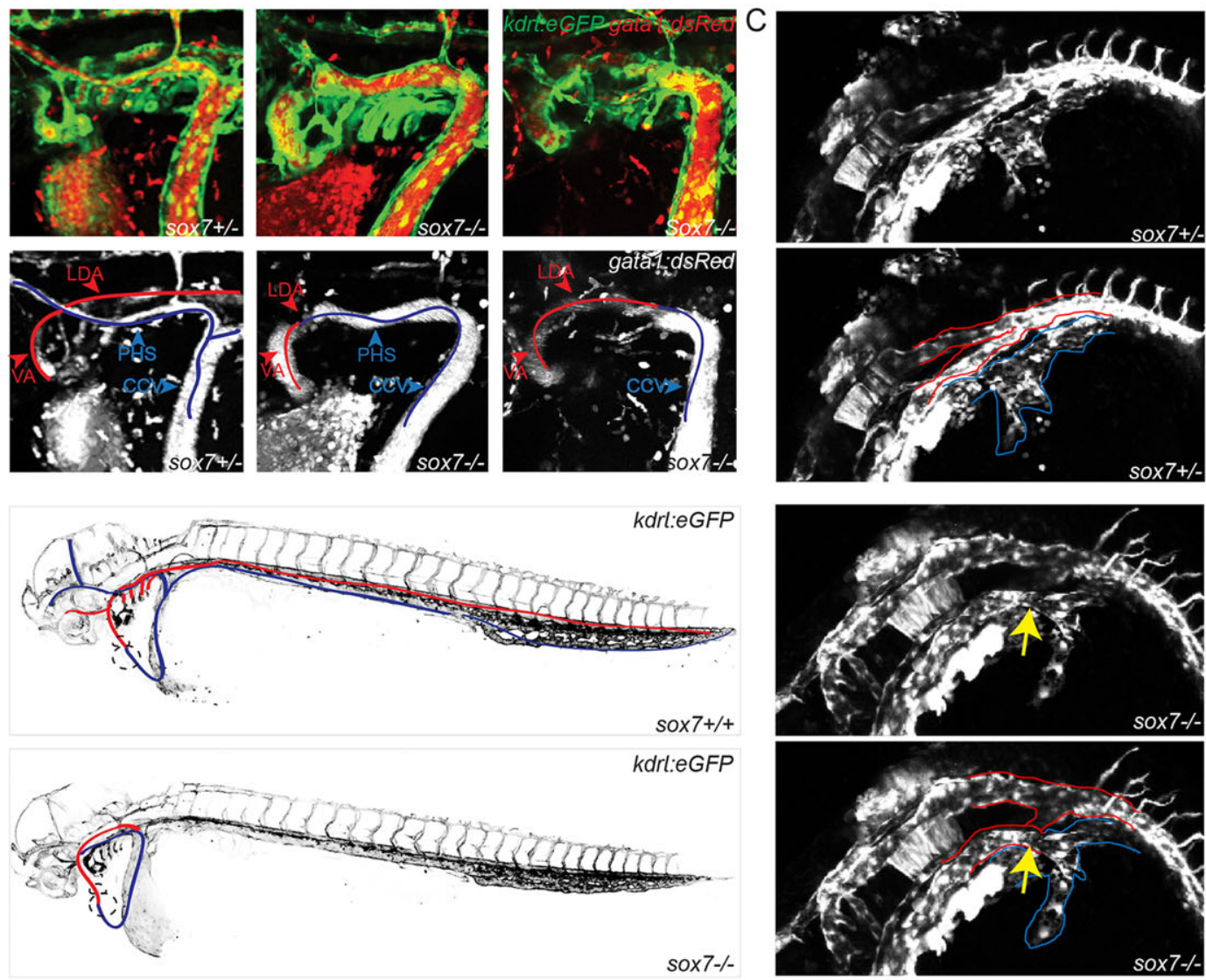

D

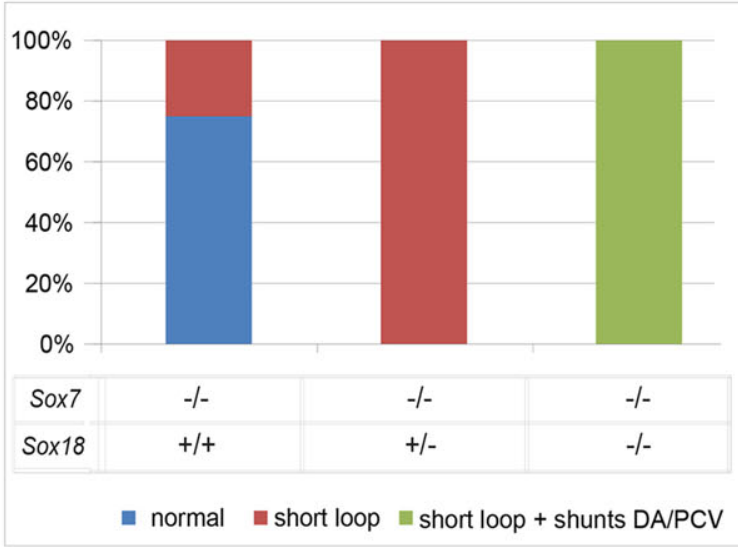

$\mathrm{E}$
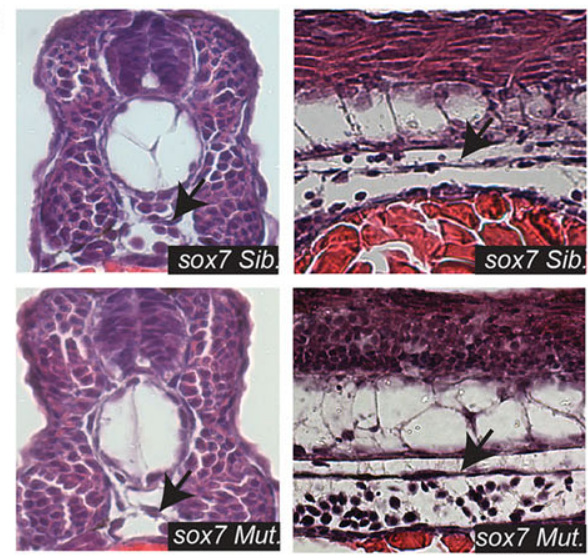

Fig. 3. sox7 mutants develop a circulatory short-loop phenotype. (A) sox $7^{\text {hu5626 }} \mathrm{kdrl}: \mathrm{eGFP}$;gata1:dsRed mutants at 2.5 dpf with shunt formation resulting in a circulatory short-loop (middle and right panel) in contrast to normal circulation in sox $7^{\text {hu5626 }}$ siblings (left panel). Middle panel depicts ectopic connection between LDA and PHS, right panel between LDA and CCV. Lower panel depicts only gata1:dsRed with detailed schematic representation of blood flow. CCV, common cardinal vein; LDA, lateral dorsal aorta; PHS, primary head sinus; VA, ventral aorta. (B) Schematic representation of normal blood flow in wild-type embryos compared with the short-circuit in sox $7^{\text {hu5626 }}$ mutants at $2.5 \mathrm{dpf}$. Dashed circles highlight the position of the heart. (A,B) Lines depict blood flow in arteries (red) and veins (blue). (C) Stills of time-lapse movies of kdrl:eGFP-positive sox 7 mutant and sibling at the time point of ectopic connection formation (see supplementary material Movies 3 and 4). ECs of the LDA (outlined by red lines) connect to ECs of the CCV (outlined by blue lines) in the sox 7 mutant at 26 hpf (yellow arrow) (D) Quantification of the phenotypes in $3 \mathrm{dpf}$ sox $7^{\text {hu5626 }}$;sox $18^{\text {hu10320 }}$ loss-of-function embryos. All sox $7^{\text {hu } 5626}$; sox $18^{\text {hu10320 }}$ double homozygous mutants have a short-loop of circulation and shunts between DA and PCV, whereas sox $7^{-1-}$ sox $18^{+/+}$and sox $7^{-1-}$ sox $18^{+/-}$embryos have no shunts between DA and PCV. The remaining, non-shown genotypic combinations of sox 7 ; sox 18 embryos all have $100 \%$ wild-type phenotype. Bars show percentages of embryos of a representative experiment (total of 77 embryos). (E) Hematoxylin and Eosin stained transverse (left panel) and sagittal (right panel) cross-sections of trunks of $2 \mathrm{dpf}$ sox $7^{\text {hu5626 }}$ siblings (top) and homozygous mutants (bottom) showing normal DA-PCV segregation in both siblings and mutants (arrows).

genetic background, $78 \%$ of sox 7 ; ephb2a double mutants developed a circulatory short-loop. This revealed a significant increase of the short-loop phenotype when combining both mutants and suggests a strong genetic interaction between ephb2a and sox7 (Fig. 5D).
Hairy/enhancer-of-split related with YRPW motif 2 (hey2) is a hes-related gene that is expressed in angioblasts from early development onwards. Previous studies have shown that mutations in hey2 (gridlock mutants) display ectopic arterial to venous connections (Weinstein et al., 1995). hey2 is a downstream target of 

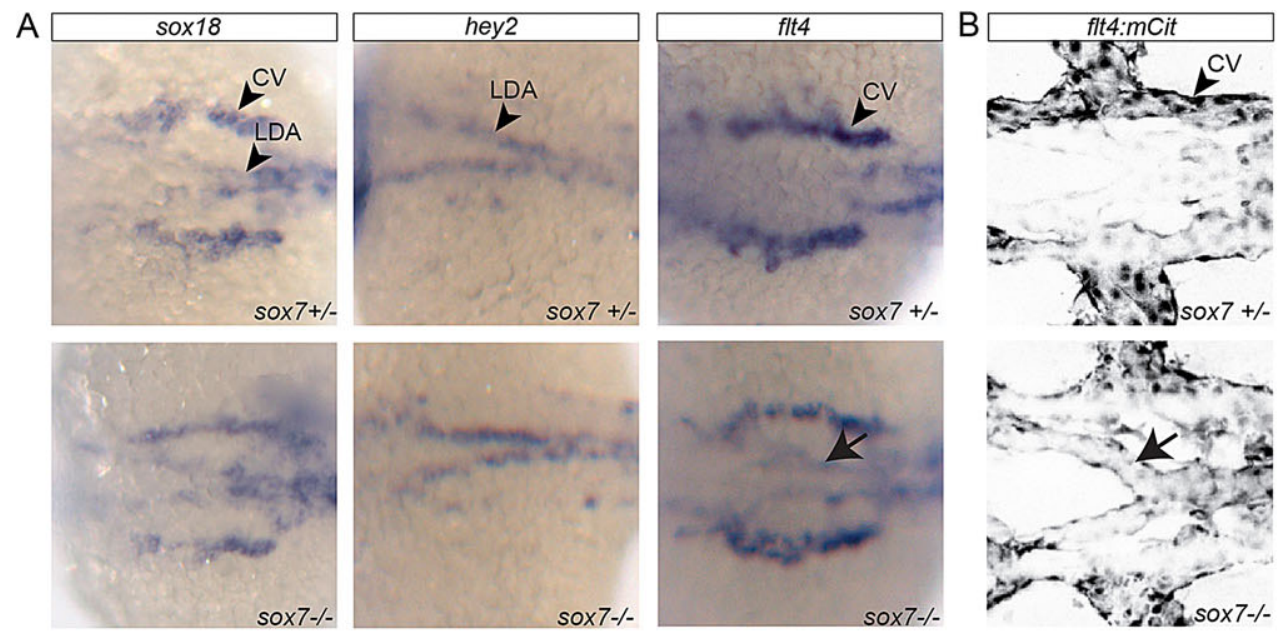

$\mathrm{C}$
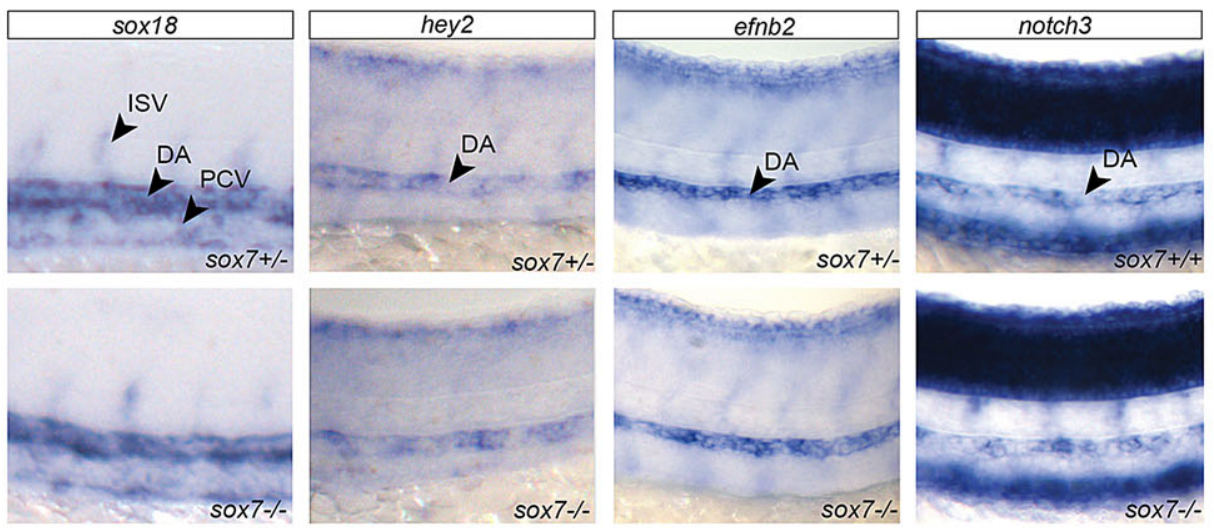

E

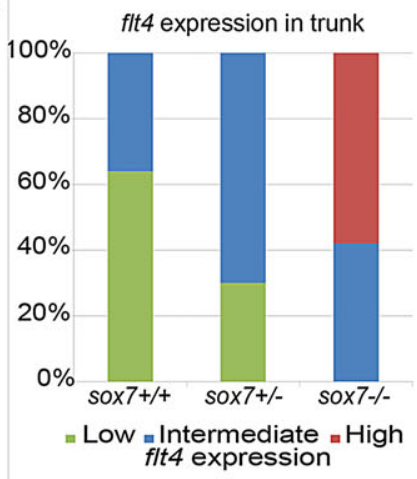

Fig. 4. flt4 expression is altered in arterial cells of sox 7 mutants.

(A-C) sox $7^{\text {hu5626 }}$ siblings (upper panel) and mutants (lower panel). (A) In situ hybridization for sox18, hey2 and flt4 in sox $7^{\text {hu5626 }}$ heterozygous and mutant embryos at $20 \mathrm{hpf}$ (dorsal view). sox $7^{\text {hu } 5626}$ mutants display elevated flt4 expression levels in the LDA (arrow). (B) Dorsal view of flt4:mCitrine (flt4:mCit)-positive sox $7^{\text {hu5626 }}$ heterozygous and homozygous mutant at $26 \mathrm{hpf}$ showing elevated $m$ Citrine expression in the LDA of $\operatorname{sox} 7^{\text {hu5626 }}$ mutant (arrow) compared with sox $7^{\text {hu5626 }}$ siblings. (C) Lateral view of the trunk region of sox $7^{\text {hu5626 }}$ siblings and mutants with in situ hybridization for sox18, hey2, efnb2, notch 3 and flt4 at 24-27 hpf. sox $7^{\text {hu5626 }}$ mutants display higher fit4 expression in DA and ISVs (arrows) compared with siblings. (D) Quantification of 20-24 hpf embryos based on their flt4 expression levels (in situ hybridization) in the LDA. Seventy-three percent of sox $7^{\text {hu5626 }}$ mutants have high expression of fIt4 in LDA region at $20 \mathrm{hpf}$ (total of 30 embryos).

(E) Quantification of 24-27 hpf embryos based on their flt4 expression levels (in situ hybridization) in the trunk. Fifty-eight percent of sox $7^{\text {hu5626 }}$ mutants have elevated flt4 expression, which was never observed in siblings (total of 57 embryos). (L)DA, (lateral) dorsal aorta; (P)CV,

(posterior) cardinal vein; ISV, intersegmental vessel.
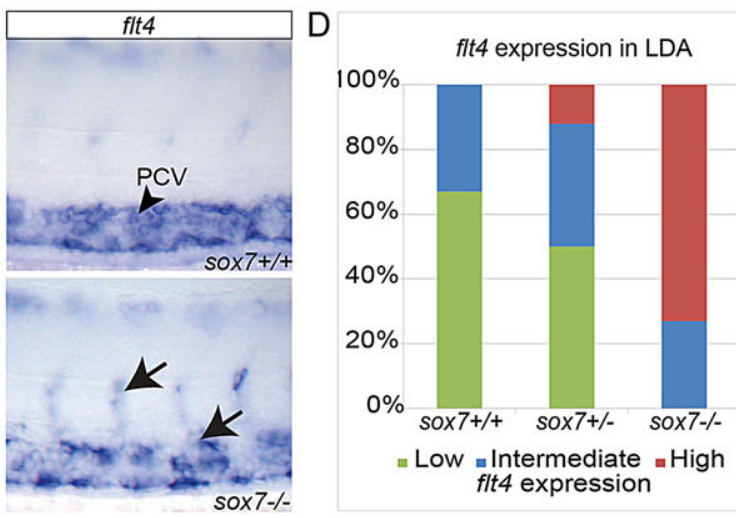

the Notch signaling pathway and participates in arterial cell-fate specification (Nakagawa et al., 2000). Injections of a hey2morpholino (MO) recapitulated the sox 7 loss-of-function phenotype in our hands with lack of blood flow in the trunk and a circulatory short-loop near the heart. To investigate a possible connection between sox 7 and hey2, we titrated down the injected amounts of hey 2 $\mathrm{MO}$ so that only minor vascular defects in wild-type embryos were evident upon injection ( $8 \%$ with $0.3 \mathrm{ng}$ ) (Fig. 6A). When injected into the offspring of a sox $7^{+/-}$in-cross, we observed an increase in the number of embryos displaying the short-loop (37\% with $0.3 \mathrm{ng}$ hey 2 $\mathrm{MO}, 5 \%$ in un-injected so $x 7^{+/-}$in-cross) (Fig. 6A). To confirm this observation, we generated hey 2 TALEN constructs targeting exon 2 upstream of the two important domains: the basic helix-loop-helix (bHLH) DNA-binding domain and the Orange domain (which confers specificity among the hairy/enhancer-of-split family) (Fig. 6B). Transient hey2 TALEN mRNA injections also resulted in the same specific circulatory defects in a subset of embryos, a phenotype that could further be reconfirmed in stable genetic hey 2 mutant embryos, again mimicking the loss of $\operatorname{sox} 7$ (data not shown). When transiently injected into a $\operatorname{sox} 7^{+/-}$in-cross the hey2 TALEN mRNA resulted in a moderate increase of the short-circuit penetrance (Fig. 6C), thereby confirming the MO data and providing independent support for the notion that both genes function together in arterial specification.

\section{Increased arterial Notch signaling suppresses the vascular defects in sox7 mutants}

Recently, in vitro studies reported that SoxF transcription factors can bind the arterial-specific enhancer of the notch ligand dll4 (Sacilotto et al., 2013). As Hey2 and Efnb2a/b have been suggested to act downstream of Notch signaling (Lawson et al., 2001) and as we show here that sox 7 genetically interacts with both factors, we 
A

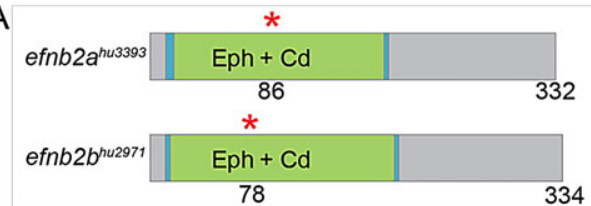

B

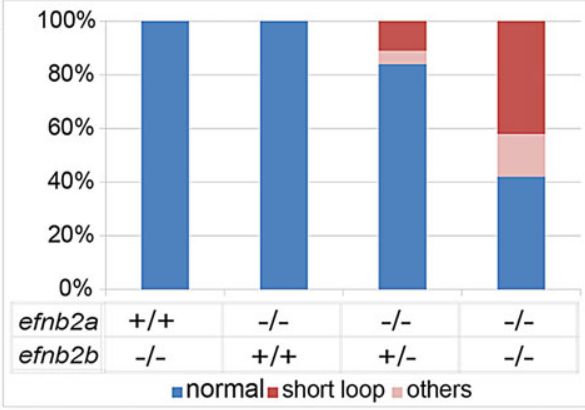

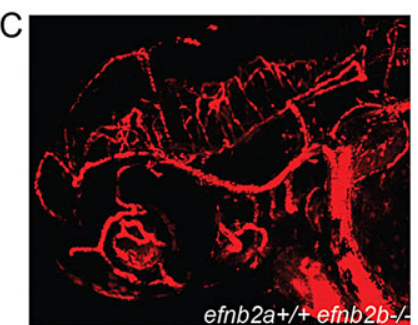
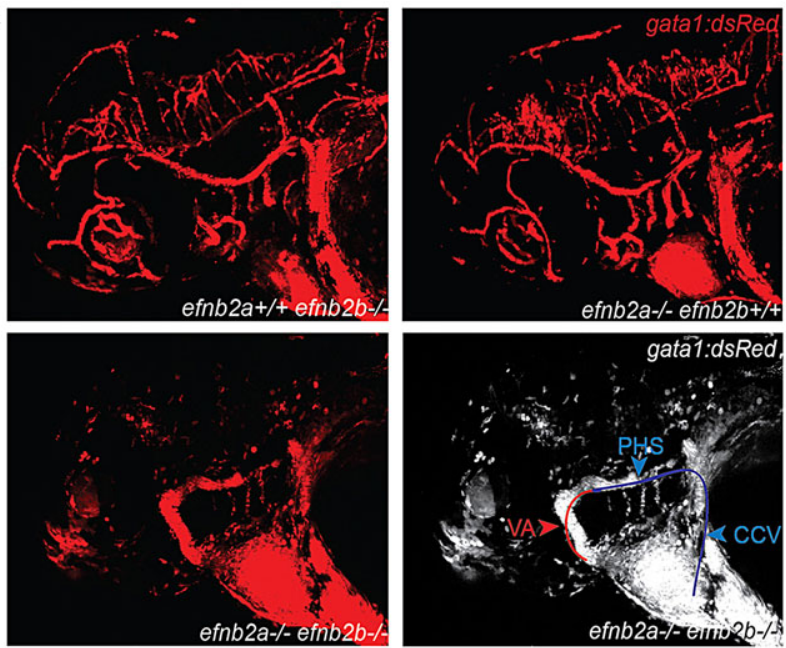

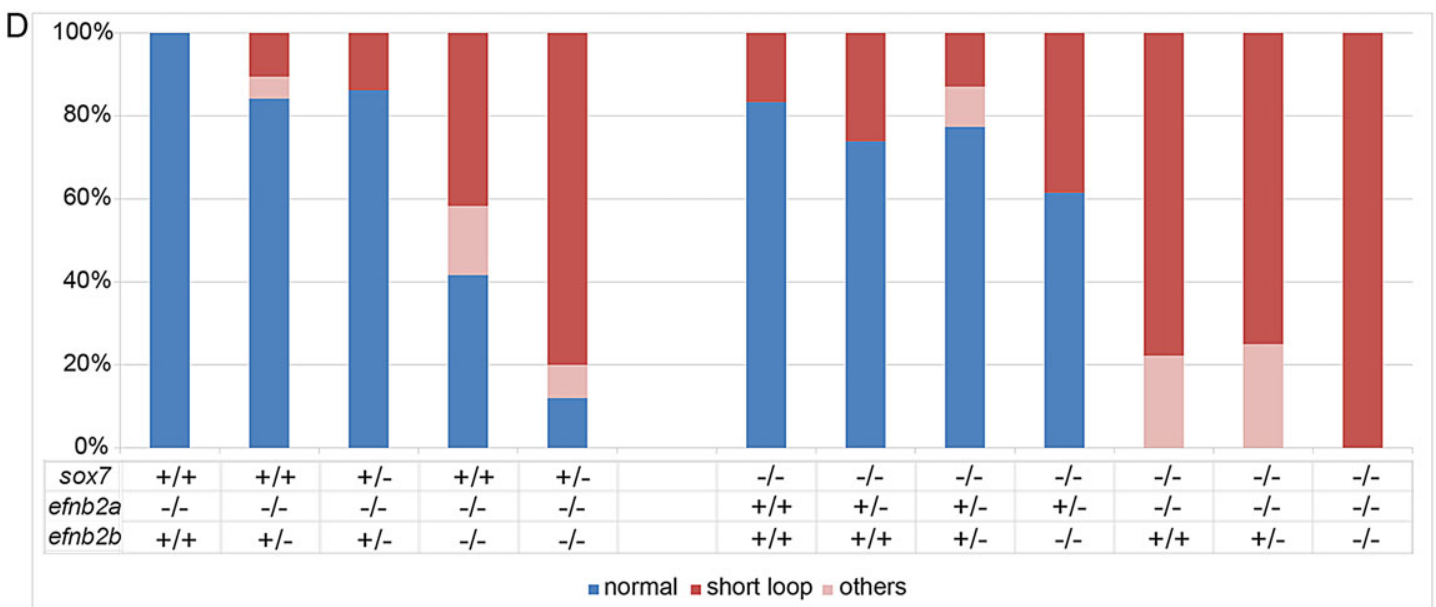

Fig. 5. efnb2a;efnb2b double mutants develop a circulatory short-circuit phenotype and sox7 genetically interacts with efnb2a. (A) Schematic diagram of efnb2a ${ }^{\text {hu} 3393}$ and efnb2 $b^{\text {hu2971 }}$ alleles with premature stop codons (red asterisks) at amino acids 86 and 78 , respectively. Eph, ephrin domain (green), Cd, cupredoxin domain (blue). (B) Quantification of efnb2a ${ }^{\text {hu} 3393}$ and $e f n b 2 b^{\text {hu2971 }}$ single and double mutant phenotypes revealed circulatory short-looping in $11 \%$ of efnb2a $a^{\text {hu } 3393-l-} ;$ efnb $2 b^{\text {hu2971+l- }}$ embryos and a significant increase of short-looping in $42 \%$ of double homozygous mutants (Student's $t$-test, $P=0.003$ compared with efnb2a $a^{\text {hu3393-l- }}$;efnb2 $b^{\text {hu} 2971+/-}$ ). The genotype combinations not shown all displayed $100 \%$ wild-type phenotype. Bars represent combined results of five independent experiments (total of 128 embryos). (C) Blood circulation in gata1:dsRed-positive efnb2a ${ }^{\text {hu } 3393}$; efnb2 $2 b^{\text {hu2971 }}$ single and double mutant embryos at $2.5 \mathrm{dpf}$. Note normal circulation in single mutants (upper panel) and short-loop phenotype in efnb2a $a^{\text {hu} 3393}$; efnb2 $2^{\text {hu2971 }}$ double mutants (lower panel). Schematic representation of blood flow in double mutants (lower right panel). Lines depict blood flow in arteries (red) and veins (blue). VA, ventral aorta; PHS, primary head sinus; CCV, common cardinal vein. (D) Combinations of sox $7^{\text {hu} 5626}$, efnb2a ${ }^{\text {hu} 3393}$ and efnb2 $b^{\text {hu}}{ }^{2971}$ loss-of-function alleles result in increasingly severe effects on the circulatory system. All sox $7^{\text {hu5626 }}$; efnb2a hu3393 double homozygous mutant embryos exhibit a significant (Student's $t$-test, $P=0.002$ ) increase in the penetrance of the short-circuit phenotype compared with the only partially penetrant $\operatorname{sox} 7^{\text {hu } 5626}$ single mutants or the efnb2a ${ }^{\text {hu } 3393}$ single mutants lacking the short-loop defect completely. The genotype combinations not shown all displayed $100 \%$ wild-type phenotype. Bars represent combined results of five independent experiments (total of 507 embryos). (B,D) Embryos were analyzed at $2.5 \mathrm{dpf}$. Phenotypes classified as 'others' are edema, total lack of circulation and/or shunts in trunk region.

wondered whether the arterial defects in sox7 mutants are a consequence of altered Notch signaling levels. We increased Notch signaling levels in arterial ECs by expressing a UAS construct encoding the Notch1 intracellular domain (NICD) (Scheer and Campos-Ortega, 1999) under the control of a newly established, arterial-specific dll4 BAC-transgenic line, the expression of which did not appear to be altered in sox 7 mutant embryos (supplementary material Fig. S5; data not shown). We found that dll4: Gal4FF;UAS: NICD expression in sox 7 mutants significantly rescued the shortloop phenotype compared with sox7 mutants lacking the UAS: NICD construct $(3 \%$ versus $27 \%$ within the same genetic background; $n=300$ embryos Fig. 6D). This demonstrates that elevated levels of arterial Notch1 signaling are sufficient to suppress the vascular defects in sox7 mutants providing direct in vivo evidence for the notion that Sox 7 acts upstream of Notch1 signaling in arterial specification.

\section{DISCUSSION}

Here, we report for the first time a detailed analysis of a genetic sox 7 loss-of-function model. sox 7 zebrafish mutants display highly specific, locally restricted defects within the vasculature. As sox 7 is expressed in all ECs, this suggests redundant roles for SoxF family members and a scenario where Sox18 (and possibly Sox17) can partially compensate for the loss of sox 7 in most vascular beds. The notion of redundancy is further supported by sox 18 zebrafish mutants not showing abnormalities during early vascular development (van Impel et al., 2014), while combined sox 7 and sox18 loss-of-function situations display rather comprehensive 

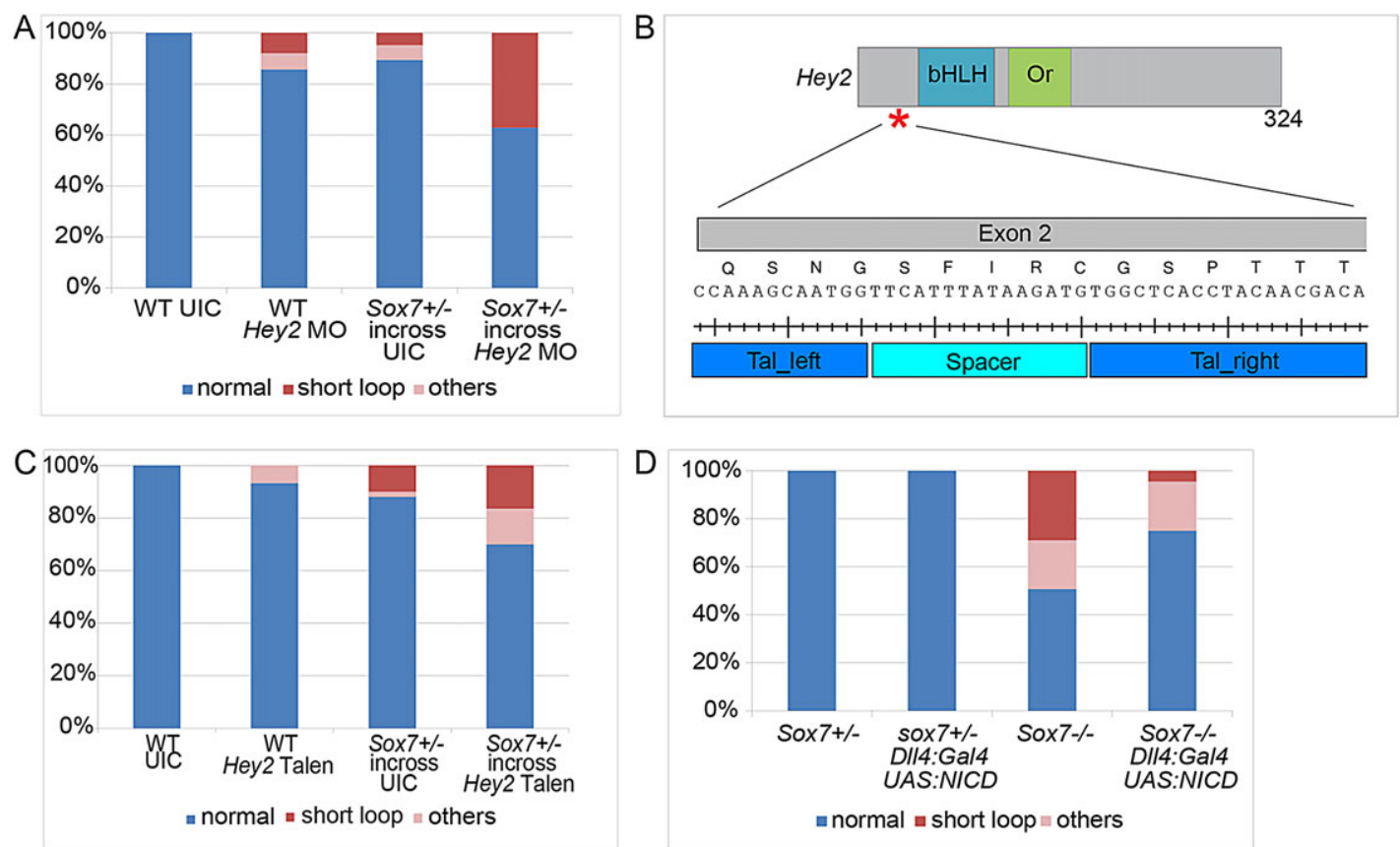

Fig. 6. sox7 synergizes with hey2 and functions upstream of Notch ICD in LDA development. (A) The partially penetrant short-loop phenotype of both sox $7^{\text {hu5626 }}$ mutants $(n=104)$ and hey 2 morphants $(0.3 \mathrm{ng}, n=63)$ is exacerbated when the hey $2 \mathrm{MO}$ is injected into a sox $7^{\text {hu5626+/- }}$ in-cross ( $\left.n=35\right)$. Bars show the percentage of embryos from a representative experiment. (B) Schematic diagram of Hey2, indicating the TALEN target site in exon 2, which is upstream of the coding region of the basic helix-loop-helix (bHLH) DNA-binding domain and the orange (Or) domain. (C) Transient injections of 10 pg per embryo hey2 TALEN mRNA into wild-type and sox $7^{\text {hu}}{ }^{5626}$ heterozygous in-cross. There is a moderate enhancement of penetrance of the short-loop phenotype in sox $7^{\text {hu}}$ 5626 in-cross injected with hey2 TALEN ( $n=140$ embryos) compared with un-injected sox $7^{\text {hu5626 }}$ in-cross ( $n=100$ embryos) and hey2 TALEN injections into wild-type embryos ( $n=90$ embryos). (D) Arterial specific UAS:NICD overexpression using the dll4:Gal4 driver line significantly (Student's $t$-test, $P=0.004$ ) rescues the short-loop circulatory phenotype in sox 7 mutants: short-loop phenotype in $27 \%$ of mutants without NICD overexpression ( $n=69$ embryos) and in $3 \%$ with NICD overexpression ( $n=44$ embryos). Bars represent pooled embryos of three independent experiments (total of 300 embryos). (A,C,D) Embryos were analyzed at $2.5 \mathrm{dpf}$. Phenotypes classified as 'others' are edema, total lack of circulation and/or shunts in trunk region.

defects, with multiple shunts between the DA and PCV in the trunk (Cermenati et al., 2008; Herpers et al., 2008; Pendeville et al., 2008) (and this study, Fig. 3D). In addition, no ectopic connections between the DA and PCV were detected in $\operatorname{sox} 7^{-1-}$;sox $18^{+/-}$ embryos, whereas the short loop of circulation is present, suggesting that one copy of sox 18 (possibly in conjunction with sox17 function) is able to establish normal DA-PCV segregation in the trunk of the zebrafish. However, we showed that the additional loss of one sox 18 allele enhances the penetrance of the short-loop phenotype in sox7 mutants, suggesting a partially overlapping function of both transcription factors also in this specific part of the arterial compartment. However, why these compensatory mechanisms do not fully prevail in the LDA remains enigmatic and suggests a unique regulation within the LDA cells. Although the DA is formed by migration of medial angioblasts originating from the posterior lateral plate mesoderm towards the midline, the LDA is formed by two distinct populations of medial angioblasts from the anterior and posterior lateral plate mesoderm that migrate towards each other and eventually connect (Siekmann et al., 2009). In addition, the initiation of LDA formation is slightly later (around 22-somite stage) compared with the formation of the DA (around 20-somite stage) (Siekmann et al., 2009), indicating that the developmental program controlling LDA formation differs from the one in other arterial ECs.

In our analysis of arterio-venous markers, only flt4 levels appeared to be changed to a detectable level, while differential expression of other arterial and/or venous markers in sox 7 mutants could not be observed. This could theoretically be explained by very specific and direct signaling between sox 7 and flt4. It seems more likely, however, that the process in question is modulated by a delicate balance of genetic interactions, where very subtle changes in expression level (too subtle to detect by in situ hybridization) are sufficient for severely disturbing arterio-venous patterning. This would also explain why we did not find differential expression of arterial markers such as the Notch target genes in sox 7 mutants, even though we demonstrate here that Sox7 can act upstream of Notch. Furthermore, the changes in expression can be highly timerestricted, leading to differential expression in only a short time interval. Although our in situ hybridization analysis was performed at $30 \mathrm{~min}$ intervals (between 20 and $30 \mathrm{hpf}$ ), it is still possible that differential expression (possibly also only at the protein level) in even a shorter time-interval can lead to severe disturbances in $\mathrm{AV}$ patterning.

Like all other Vegf receptors, flt4 is initially expressed widely throughout all vascular beds, with its expression in the trunk becoming restricted to venous cells after $26 \mathrm{hpf}$ in zebrafish (Hogan et al., 2009b). Hence, a plausible scenario is that the ectopic flt4 expression in arterial cells of sox 7 mutants results from a lack of proper downregulation of flt4 specifically in these arterial cells. Flt4 and its ligands VegfC and VegfD have been shown to be involved in endothelial cell sprouting and migration (Karkkainen et al., 2004; Tammela et al., 2008; Hogan et al., 2009b; Villefranc et al., 2013). It is therefore a possibility that the elevated flt4 levels in the arterial cells of the sox 7 mutants result in a disrupted balance between Flt4 signaling levels in ECs of the LDA and the surrounding venous vessels, which in conjunction with a potential mis-regulation of other arterial and venous patterning genes causes misguidance of ECs and the formation of ectopic shunts. 
On the other hand, previous work in mice and cultured ECs demonstrated that Efnb2 can mediate the internalization and thereby contribute to the signaling activity of Flt4 (Wang et al., 2010). As we show here that efnb2 functions together with $\operatorname{sox} 7$ and both genes are essential for proper LDA development, the lack of efnb2 (and potentially indirectly also of sox7) could also result in reduced internalization of Flt4 and therefore to a decrease in its signaling potential. It is plausible that the embryo would react to such a decrease in Flt4 signaling activity by increasing the expression of the receptor. Hence, the elevated mRNA levels of flt 4 in sox 7 mutants could also hint towards a compensation mechanism rather than being a sign of increased Flt4 signaling levels in LDA cells. This would make the elevated flt 4 mRNA levels a consequence of the $\operatorname{sox} 7$ defects instead of being the actual cause of the shunt formation.

The very specific circulatory defects in sox 7 , hey2 and efnb2 mutants, together with the genetic interaction study presented here strongly suggest a synergistic relationship between sox 7 and both hey 2 and efnb2. Whether these proteins function in one pathway or in parallel pathways that synergize to function in arterial development remains unclear at present. We suggest a model where Sox7 (and in a partially redundant manner Sox18) acts upstream of the Notch signaling pathway while controlling flt4 expression and potentially other arterio-venous patterning genes, and functioning in conjunction with Hey2 and Efnb2 in arterio-venous specification.

The locally restricted effect of the $\operatorname{sox} 7$ mutation indicates a level of redundancy in terms of the activity of SoxF family members to a degree that leads, for example, to AV defects in the trunk only upon severe reduction of both Sox7 and Sox 18 (Herpers et al., 2008; this study). All endothelial cells appear to be able to compensate for the loss of Sox 18 function (van Impel, 2014), and most endothelial cells can compensate for loss of Sox7 (this study) - the only exception being the endothelial cells of the LDA, which crucially depend on Sox7 function. It is unclear at present why the LDA represents a vascular bed that displays this specific requirement, but the occurrence of the same locally restricted phenotype in three different mutant scenarios indeed points towards unique features in the genetic control of endothelial cell behavior within the LDA.

\section{MATERIALS AND METHODS Zebrafish}

Zebrafish were maintained under standard husbandry conditions according to the rules of the Animal Experimentation Committee (DEC) of the KNAW. Transgenic lines used were $\operatorname{Tg}(f l i l a: e G F P)^{\mathrm{y} 1}$ (Lawson and Weinstein, 2002), $T g(k d r l: e G F P)^{\text {s843 }}$ (Jin et al., 2005), $T g$ (gata1: $d s R e d)^{\text {sd2 }}$ (Traver et al., 2003), $T g(k d r l: H R A S-m C h e r r y)^{s 916}$ (Hogan et al., 2009a), $\operatorname{Tg}(\text { flt4:mCitrine })^{\text {hut135 }}$ (van Impel et al., 2014), $\operatorname{Tg}($ Sox17:eGFP) (Mizoguchi et al., 2008) and Tg(UAS:myc-NICD) (Scheer and CamposOrtega, 1999). The Tg(dll4:Gal4FF ${ }^{\text {hul0049 }}$ ) line was generated from BAC $\mathrm{CH} 211-19 \mathrm{M} 2$ following standard recombineering procedures (Bussmann and Schulte-Merker, 2011). The sox $18^{\text {hul } 10320}$ allele has been previously described (van Impel et al., 2014)

\section{Genotyping, morpholinos and TALEN constructs}

Genotyping of $\operatorname{sox} 7, e f n b 2 a$ and $e f n b 2 b$ was performed by KASPAR with primers listed in supplementary material Table S1. The hey2 MO used was 5'-CGCGCAGGTACAGACACCAAAAACT-3' (GeneTools) (Zhong et al., 2001); $0.3 \mathrm{ng}$ of hey $2 \mathrm{MO}$ was used for suboptimal effect. hey 2 TALEN binding sites are: TAL1, 5'-TGTCGTTGTAGGTGAGCCA-3'; TAL2, 5'-TATTCCCAGCCAAAGCAATGG-3'; primers are mentioned in supplementary material Table S1. Talen constructs were generated as described previously (Cermak et al., 2011; Bedell et al., 2012).

\section{Histology and in situ hybridization}

In situ hybridization and immunohistochemistry were performed as described previously (Schulte-Merker, 2002). For immunohistochemistry, the embryos were stained with rabbit anti-GFP 1:1000 and anti-rabbit Alexa 488 1:1000 (Invitrogen), or stained with Hematoxylin and Eosin. The embryos were paraffin-embedded and sectioned $(7 \mu \mathrm{m})$, after which they were analyzed with a Leica TCS SPE. For in situ hybridization we used previously described hey2 (Zhong et al., 2000), sox 18 (Herpers et al., 2008), efnb2 (Chan et al., 2001), notch3 (Lawson et al., 2001) and flt4 (Bussmann et al., 2007) probes.

\section{Microscopy}

Confocal imaging was performed on live embryos embedded in $0.5 \%$ low melting point agarose (Invitrogen) with MS222 (0.04\%) and 1-phenyl-2thiourea (PTU $0.003 \%$ ) using a Leica TCS SPE. Bright-field pictures and movies were acquired on a Zeiss Axioplan microscope and a Leica TCS SPE, respectively. Images were processed using Adobe Photoshop CS5.1 and Fiji (http://fiji.sc/Fiji).

\section{Micro-angiography}

Micro-angiography was performed by injecting rhodamine dextran $(10 \mathrm{mg} / \mathrm{ml})$ into the PCV of MS222-anaesthetized embryos, as described previously (Weinstein et al., 1995). Embryos were mounted in 0.5\% low melting-point agarose and imaged with a Leica TCS SPE.

\section{RT-PCR analysis}

sox 7 siblings and sox 7 mutants were first selected based on the short-loop phenotype and pooled separately $(n=10$ embryos/pool) at 2 dpf. cDNA was synthesized from RNA with primers listed in supplementary material Table S1.

\section{Acknowledgements}

We thank J. Peterson-Maduro for technical assistance, A. de Graaff and the Hubrecht Imaging Centre for supporting the imaging, J. Korving and $\mathrm{H}$. Begthel for supporting the histology, J. Bakkers for the sox17:eGFP reporter line, J. den Hertog for the hey2 morpholino, and B. Tembuyser and A. Luttun for the nr2f2 in situ hybridization probe.

\section{Competing interests}

The authors declare no competing or financial interests.

\section{Author contributions}

D.M.A.H. performed the experiments, analyzed the new sox7, hey2 and efnb2 mutants, and wrote the manuscript. J.B. engineered the dll4:gal4FF BAC. A.v.I. injected and established the dII4:gal4FF;UAS:RFP transgenic line, and generated the flt4:mCitrine reporter line. A.U. analyzed the efnb2 mutants. D.M.A.H., A.v.I. and S.S.-M. designed the experiments and edited the manuscript. H.J.D. and S.S.-M. conceived and supervised the study.

\section{Funding}

This work was supported by the Dutch Scientific Organization [NWO VIDI 1776325] the KNAW and the Deutsche Forschungsgemeinschaft (DFG), Cells-in-Motion Cluster of Excellence [EXC $1003-\mathrm{CiM}]$

\section{Supplementary material}

Supplementary material available online at

http://dev.biologists.org/lookup/suppl/doi:10.1242/dev.117275/-/DC1

\section{References}

Achen, M. G., Jeltsch, M., Kukk, E., Makinen, T., Vitali, A., Wilks, A. F., Alitalo, K. and Stacker, S. A. (1998). Vascular endothelial growth factor D (VEGF-D) is a ligand for the tyrosine kinases VEGF receptor 2 (FIk1) and VEGF receptor 3 (FIt4). Proc. Natl. Acad. Sci. USA 95, 548-553.

Bedell, V. M., Wang, Y., Campbell, J. M., Poshusta, T. L., Starker, C. G., Krug R. G., II, Tan, W., Penheiter, S. G., Ma, A. C., Leung, A. Y. H. et al. (2012). In vivo genome editing using a high-efficiency TALEN system. Nature 491 114-118

Brouillard, P. and Vikkula, M. (2007). Genetic causes of vascular malformations. Hum. Mol. Genet. 16, R140-R149.

Bussmann, J. and Schulte-Merker, S. (2011). Rapid BAC selection for tol2mediated transgenesis in zebrafish. Development 138, 4327-4332. 
Bussmann, J., Bakkers, J. and Schulte-Merker, S. (2007). Early endocardia morphogenesis requires Scl/Tal1. PLoS Genet. 3, e140.

Cermak, T., Doyle, E. L., Christian, M., Wang, L., Zhang, Y., Schmidt, C., Baller, J. A., Somia, N. V., Bogdanove, A. J. and Voytas, D. F. (2011). Efficient design and assembly of custom TALEN and other TAL effector-based constructs for DNA targeting. Nucleic Acids Res. 39, e82.

Cermenati, S., Moleri, S., Cimbro, S., Corti, P., Del Giacco, L., Amodeo, R., Dejana, E., Koopman, P., Cotelli, F. and Beltrame, M. (2008). Sox18 and Sox7 play redundant roles in vascular development. Blood 111, 2657-2666.

Chan, J., Mably, J. D., Serluca, F. C., Chen, J.-N., Goldstein, N. B., Thomas, M. C., Cleary, J. A., Brennan, C., Fishman, M. C. and Roberts, T. M. (2001) Morphogenesis of prechordal plate and notochord requires intact Eph/ephrin B signaling. Dev. Biol. 234, 470-482.

Corada, M., Orsenigo, F., Morini, M. F., Pitulescu, M. E., Bhat, G., Nyqvist, D., Breviario, F., Conti, V., Briot, A., Iruela-Arispe, M. L. et al. (2013). Sox17 is indispensable for acquisition and maintenance of arterial identity. Nat. Commun. 4, 2609.

François, M., Caprini, A., Hosking, B., Orsenigo, F., Wilhelm, D., Browne, C. Paavonen, K., Karnezis, T., Shayan, R., Downes, M. et al. (2008). Sox18 induces development of the lymphatic vasculature in mice. Nature 456, 643-647.

Francois, M., Koopman, P. and Beltrame, M. (2010). SoxF genes: Key players in the development of the cardio-vascular system. Int. J. Biochem. Cell Biol. 42 445-448.

Gerety, S. S., Wang, H. U., Chen, Z.-F. and Anderson, D. J. (1999). Symmetrical mutant phenotypes of the receptor EphB4 and its specific transmembrane ligand ephrin-B2 in cardiovascular development. Mol. Cell 4, 403-414.

Giese, K., Cox, J. and Grosschedl, R. (1992). The HMG domain of lymphoid enhancer factor 1 bends DNA and facilitates assembly of functional nucleoprotein structures. Cell 69, 185-195.

He, S., Kim, I., Lim, M. S. and Morrison, S. J. (2011). Sox17 expression confers self-renewal potential and fetal stem cell characteristics upon adult hematopoietic progenitors. Genes Dev. 25, 1613-1627.

Herpers, R., van de Kamp, E., Duckers, H. J. and Schulte-Merker, S. (2008) Redundant roles for sox7 and sox18 in arteriovenous specification in zebrafish Circ. Res. 102, 12-15.

Hogan, B. M., Bos, F. L., Bussmann, J., Witte, M., Chi, N. C., Duckers, H. J. and Schulte-Merker, S. (2009a). Ccbe1 is required for embryonic lymphangiogenesis and venous sprouting. Nat. Genet. 41, 396-398.

Hogan, B. M., Herpers, R., Witte, M., Helotera, H., Alitalo, K., Duckers, H. J. and Schulte-Merker, S. (2009b). Vegfc/Flt4 signalling is suppressed by Dll4 in developing zebrafish intersegmental arteries. Development 136, 4001-4009.

Hosking, B. M., Muscat, G. E. O., Koopman, P. A., Dowhan, D. H. and Dunn, T. L. (1995). Trans-activation and DNA-binding properties of the transcription factor, Sox-18. Nucleic Acids Res. 23, 2626-2628.

Hudson, C., Clements, D., Friday, R. V., Stott, D. and Woodland, H. R. (1997) Xsox17alpha and -beta mediate endoderm formation in Xenopus. Cell $\mathbf{9 1}$ 397-405.

Irrthum, A., Devriendt, K., Chitayat, D., Matthijs, G., Glade, C., Steijlen, P. M., Fryns, J.-P., Van Steensel, M. A. M. and Vikkula, M. (2003). Mutations in the transcription factor gene SOX18 underlie recessive and dominant forms of hypotrichosis-lymphedema-telangiectasia. Am. J. Hum. Genet. 72, 1470-1478.

James, K., Hosking, B., Gardner, J., Muscat, G. E. O. and Koopman, P. (2003) Sox18 mutations in the ragged mouse alleles ragged-like and opossum. Genesis 36, 1-6.

Jin, S.-W., Beis, D., Mitchell, T., Chen, J.-N. and Stainier, D. Y. R. (2005). Cellular and molecular analyses of vascular tube and lumen formation in zebrafish. Development 132, 5199-5209.

Joukov, V., Pajusola, K., Kaipainen, A., Chilov, D., Lahtinen, I., Kukk, E., Saksela, O., Kalkkinen, N. and Alitalo, K. (1996). A novel vascular endothelial growth factor, VEGF-C, is a ligand for the FIt4 (VEGFR-3) and KDR (VEGFR-2) receptor tyrosine kinases. EMBO J. 15, 290-298.

Kaipainen, A., Korhonen, J., Mustonen, T., van Hinsbergh, V. W., Fang, G. H., Dumont, D., Breitman, M. and Alitalo, K. (1995). Expression of the fms-like tyrosine kinase 4 gene becomes restricted to lymphatic endothelium during development. Proc. Natl. Acad. Sci. USA 92, 3566-3570.

Kanai-Azuma, M., Kanai, Y., Gad, J. M., Tajima, Y., Taya, C., Kurohmaru, M. Sanai, Y., Yonekawa, H., Yazaki, K., Tam, P. P. et al. (2002). Depletion of definitive gut endoderm in Sox17-null mutant mice. Development 129 2367-2379.

Karkkainen, M. J., Haiko, P., Sainio, K., Partanen, J., Taipale, J., Petrova, T. V., Jeltsch, M., Jackson, D. G., Talikka, M., Rauvala, H. et al. (2004). Vascular endothelial growth factor $\mathrm{C}$ is required for sprouting of the first lymphatic vessels from embryonic veins. Nat. Immunol. 5, 74-80.
Kohli, V., Schumacher, J. A., Desai, S. P., Rehn, K. and Sumanas, S. (2013). Arterial and venous progenitors of the major axial vessels originate at distinct locations. Dev. Cell 25, 196-206.

Lawson, N. D. and Weinstein, B. M. (2002). In vivo imaging of embryonic vascular development using transgenic zebrafish. Dev. Biol. 248, 307-318.

Lawson, N. D., Scheer, N., Pham, V. N., Kim, C. H., Chitnis, A. B., CamposOrtega, J. A. and Weinstein, B. M. (2001). Notch signaling is required for arterialvenous differentiation during embryonic vascular development. Development 128, 3675-3683.

Lawson, N. D., Vogel, A. M. and Weinstein, B. M. (2002). sonic hedgehog and vascular endothelial growth factor act upstream of the Notch pathway during arterial endothelial differentiation. Dev. Cell 3, 127-136.

Mizoguchi, T., Verkade, H., Heath, J. K., Kuroiwa, A. and Kikuchi, Y. (2008) Sdf1/Cxcr4 signaling controls the dorsal migration of endodermal cells during zebrafish gastrulation. Development 135, 2521-2529.

Nakagawa, O., McFadden, D. G., Nakagawa, M., Yanagisawa, H., Hu, T. Srivastava, D. and Olson, E. N. (2000). Members of the HRT family of basic helix-loop-helix proteins act as transcriptional repressors downstream of Notch signaling. Proc. Natl. Acad. Sci. USA 97, 13655-13660.

Pendeville, H., Winandy, M., Manfroid, I., Nivelles, O., Motte, P., Pasque, V., Peers, B., Struman, I., Martial, J. A. and Voz, M. L. (2008). Zebrafish Sox7 and Sox18 function together to control arterial-venous identity. Dev. Biol. 317 405-416.

Pennisi, D., Gardner, J., Chambers, D., Hosking, B., Peters, J., Muscat, G., Abbott, C. and Koopman, P. (2000). Mutations in Sox18 underlie cardiovascular and hair follicle defects in ragged mice. Nat. Genet. 24, 434-437.

Sacilotto, N., Monteiro, R., Fritzsche, M., Becker, P. W., Sanchez-Del-Campo, L. Liu, K., Pinheiro, P., Ratnayaka, I., Davies, B., Goding, C. R. et al. (2013) Analysis of DII4 regulation reveals a combinatorial role for Sox and Notch in arterial development. Proc. Natl. Acad. Sci. USA 110, 11893-11898.

Scheer, N. and Campos-Ortega, J. A. (1999). Use of the Gal4-UAS technique for targeted gene expression in the zebrafish. Mech. Dev. 80, 153-158.

Schulte-Merker, S. (2002). Looking at embryos. In Zebrafish: A Practical Approach (ed. C. Nusslein-Volhard and R. Dahm), Chapter 2, p. 39. New York, NY: Oxford University Press.

Siekmann, A. F., Standley, C., Fogarty, K. E., Wolfe, S. A. and Lawson, N. D. (2009). Chemokine signaling guides regional patterning of the first embryonic artery. Genes Dev. 23, 2272-2277.

Tammela, T., Zarkada, G., Wallgard, E., Murtomäki, A., Suchting, S., Wirzenius, M., Waltari, M., Hellström, M., Schomber, T., Peltonen, R. et al. (2008). Blocking VEGFR-3 suppresses angiogenic sprouting and vascular network formation. Nature 454, 656-660.

Traver, D., Paw, B. H., Poss, K. D., Penberthy, W. T., Lin, S. and Zon, L. I. (2003) Transplantation and in vivo imaging of multilineage engraftment in zebrafish bloodless mutants. Nat. Immunol. 4, 1238-1246.

van Impel, A., Zhao, Z., Hermkens, D. M. A., Roukens, M. G., Fischer, J. C. Peterson-Maduro, J., Duckers, H., Ober, E. A., Ingham, P. W. and SchulteMerker, S. (2014). Divergence of zebrafish and mouse lymphatic cell fate specification pathways. Development 141, 1228-1238.

Villefranc, J. A., Nicoli, S., Bentley, K., Jeltsch, M., Zarkada, G., Moore, J. C., Gerhardt, H., Alitalo, K. and Lawson, N. D. (2013). A truncation allele in vascular endothelial growth factor $\mathrm{c}$ reveals distinct modes of signaling during lymphatic and vascular development. Development 140, 1497-1506.

Wang, H. U., Chen, Z.-F. and Anderson, D. J. (1998). Molecular distinction and angiogenic interaction between embryonic arteries and veins revealed by ephrinB2 and its receptor Eph-B4. Cell 93, 741-753.

Wang, Y., Nakayama, M., Pitulescu, M. E., Schmidt, T. S., Bochenek, M. L., Sakakibara, A., Adams, S., Davy, A., Deutsch, U., Lüthi, U. et al. (2010). Ephrin-B2 controls VEGF-induced angiogenesis and lymphangiogenesis. Nature 465, 483-486.

Wat, M. J., Beck, T. F., Hernandez-Garcia, A., Yu, Z, Veenma, D., Garcia, M. Holder, A. M., Wat, J. J., Chen, Y., Mohila, C. A. et al. (2012). Mouse model reveals the role of SOX7 in the development of congenital diaphragmatic hernia associated with recurrent deletions of 8p23.1. Hum. Mol. Genet. 21, 4115-4125.

Weinstein, B. M., Stemple, D. L., Driever, W. and Fishman, M. C. (1995). Gridlock a localized heritable vascular patterning defect in the zebrafish. Nat. Med. 1 1143-1147.

Wienholds, E., Schulte-Merker, S., Walderich, B. and Plasterk, R. H. (2002) Target-selected inactivation of the zebrafish rag1 gene. Science 297, 99-102.

Zhong, T. P., Rosenberg, M., Mohideen, M.-A., Weinstein, B. and Fishman, M. C (2000). gridlock, an HLH gene required for assembly of the aorta in zebrafish Science 287, 1820-1824.

Zhong, T. P., Childs, S., Leu, J. P. and Fishman, M. C. (2001). Gridlock signalling pathway fashions the first embryonic artery. Nature 414, 216-220. 